\title{
Fluctuating Morality: The Effects of Moral Identity and Self-affirmation on Pro-Social Behavior
}

\author{
Jian $\mathrm{Nie}^{1}$, Wei Shi ${ }^{1}$, and Zixuan Tang ${ }^{2}$ \\ ${ }^{1}$ School of Culture and Social Development Studies, Southwest University, Applied Psychology, 400715 \\ ${ }^{2}$ School of Culture and Social Development Studies, Southwest University, Public Relations, 400715
}

Keywords: morality; self-affirmation; pro-social behavior

\begin{abstract}
Although the self-affirmation and self-consistency theories emphasize the maintenance of self-concept, there are differences in the way for the two theories to view the topic. Thus, the two theories differ with regard to pro-social behaviors. In this study, experiments are conducted to investigate two separate aspects of self-concept, moral identity and self-affirmation. The findings reveal that the degree of moral dissonance experienced by the participants is regulated by one's moral identity through self-affirmation and that the regulation affects subsequent donation behavior. This study quantifies the degree of moral dissonance and preliminarily addresses the divergences between the self-affirmation and self-consistency theories with respect to pro-social behaviors.
\end{abstract}

\section{Introduction}

In the process of moral self-regulation, individuals aim to maintain their moral identity at an appropriate level(Marquardt, Gantman, Gollwitzer, \& Oettingen, 2016; Sachdeva, Iliev, \& Medin, 2009). However, there are theoretical divergences with respect to regulation methods. The self-consistency theory focuses on the issues that trigger self-incongruence, whereas the self-affirmation theory deems any method that determines self-integrity from an overall approach feasible(Steele, Spencer, \& Lynch, 1993; Thibodeau \& Aronson, 1992). However, the self-consistency theory finds that individuals engage in behavior to compensate for the damaged sense of self, thereby engaging in more pro-social behavior. In this study, we performed experiments that demonstrated the effects of self-affirmation and moral identity on pro-social behaviors, thus investigating the divergences between the two theories regarding pro-social behavior through the different domains of self-affirmation (i.e., related or unrelated to morality).

\section{Literature Review}

The moral self. Monin and Jordan find that moral identity is based on the effects of personality on the moral self. The moral self is relatively stable, although differences exist among individuals. Reed, Aquino and Levy conducted experiments that used particular wordings to activate the moral identities of their participants. They found that participants with high levels of activated moral identity would contribute more time to charitable organizations than participants with low levels of activated moral identity(Reed, Aquino, \& Levy, 2007). Therefore, the varying levels of moral identity among individuals might be a key factor in fluctuations of individual morality.

Studies on the moral self from a social psychology viewpoint have been able to quantify the changes in one's moral self under different circumstances. Scholars have coined the term moral self-image (MSI) based on moral identity. MSI is a scalable moral self-concept of individuals related to their typical moral traits(Jordan, Leliveld, \& Tenbrunsel, 2015). MSI primarily involves the perceived moral traits of individuals demonstrated personally at a specific point in time, and the changes in one's moral self under different circumstances are quantifiable. Therefore, MSI may be used as a benchmark to assess the moral dissonance of individuals. The MSI scale formulated by Jordan et al. (2015) can serve as a supporting tool for the dynamic measurement of changes in the moral selves of individuals of different moral identities under experimental circumstances. 


\section{Experiment Materials and Method}

\subsection{Participants}

Participants were 190 undergraduates (88 males, 102 female) at the Southwest University who participated in the study for course credit. Based on the moral identity measure scores, the participants who scored in the lowest $27 \%$ and the participants who scored the highest $27 \%$ were grouped separately. The participants in the low-score and high-score groups were invited to participate in the main experiment. A total of 85 valid data samples were collected. The sample group consisted of 37 males and 48 females, with an average age of 19.19 years (from 18 to 20 years, $\mathrm{SD}=1.13)$.

\subsection{Procedure}

Fifteen hours prior to the experiment, the participants were requested to complete the MSI scale ( 9 items; $\alpha=0.94$ ) and the Moral Identity Measure (MIM) (10 items; $\alpha=0.98)$. Based on the MIM score, the participants who scored the highest (27\%) and the participants who scored the lowest (27\%) were divided into the high moral identity group and the low moral identity group. On arriving at the laboratory for the main experiment, the participants were informed that the experiment consisted of two sections, a memory test and a cognitive test. All tests were taken on computers. The first section of the experiment was based on a study by Sachdeva et al. that found that changes in the evaluation of one's moral self could only be triggered when an individual recalls past moral behavior of his or her own (Sachdeva et al., 2009). Therefore, we requested the participants to recall and write down a past immoral behavior that they performed. For the control of the recollection period, we referred to a study by Conway et al. on construal levels(Conway \& Peetz, 2012). To prevent moral fluctuation from interfering with the experiment findings as the participants recalled a recent immoral behavior, we asked the participants to recall an event that occurred within the past year.

\section{Measurements}

In refer to the practice of Mulder and Aquino (2013) study, we divided the respondents into a high moral identity group and a low moral identity group based on the internalization sub-dimension, whereby the respondents with the lowest and highest internalization sub-dimension scores (27\%) were classified into the groups.

We used the MSI scale and referred to the paradigms of Jordan et al.(Jordan et al., 2015) The levels of moral dissonance faced by the participants are represented by the differences between the MSI scores on the tests administered 15 hours prior to the commencement of the main experiment and the MSI test scores collected on completion of the experiment.

A number of studies have demonstrated that individuals find it easy to spend unexpected income but difficult to spend hard-earned income(Henderson \& Robert, 1992; Xiaofu, Zhaojing, Fei, \& Ying, 2014). Therefore, we asked the participants to make a donation based on the payment received from participating in this study. To eliminate the effects of mental accounting on donation behavior, we did not inform the participants of the amount of payment they would receive. We only informed them that they would receive payment and asked them how much of the payment they would be willing to donate to assist orphans living in urban areas.

\section{Empirical Results}

\subsection{Experiment analysis and demographic variables}

There were no significant one-way analysis of variance (ANOVA) findings for the MSI scores according to gender $\left(\mathrm{F}_{1,83}=0.61, \mathrm{P}=0.44\right)$. There were also no significant one-way ANOVA findings for pro-social behavior by gender $\left(\mathrm{F}_{1,83}=2.78, \mathrm{P}=0.01\right)$. These findings indicate that gender did not affect pro-social behavior and MSI scores. Regarding the ANOVA conducted on the participants' self-evaluation of the degree of immorality of their recalled immoral behaviors, there were no 
significant findings for gender $\left(\mathrm{F}_{1,83}=0.42, \mathrm{P}=0.52\right)$, moral identity group $\left(\mathrm{F}_{1,83}=0.06, \mathrm{P}=0.81\right)$ and self-affirmation group $\left(\mathrm{F}_{1,82}=0.36, \mathrm{P}=0.70\right)$. These outcomes indicate that there were no differences regarding the degree of immorality of the recalled immoral behavior.

\subsection{Analysis of moral dissonance}

In the two-way between-subjects ANOVA, self-affirmation and moral identity were independent variables, while the difference between the first and second MSI scores was a dependent variable (means and standard deviations of the MSI scores are presented in Table 1). The findings indicated that the main effect of self-affirmation was significant $\left(\mathrm{F}_{2,79}=8.28, \mathrm{P}=0.001\right.$, partial $\left.\eta^{2}=0.16\right)$ and that the main effect of moral identity was insignificant $\left(\mathrm{F}_{1,79}=0.02, \mathrm{P}=0.88\right.$, partial $\left.\eta^{2}<0.001\right)$, as reported in Table 2. The interactive influence was significant $\left(\mathrm{F}_{2,79}=5.67, \mathrm{P}=0.005\right.$, partial $\left.\eta^{2}=0.11\right)$. A simple effect analysis revealed significant discrepancies in the MSI score differences between individuals with high moral identity and individuals with low moral identity under the condition that self-affirmation and morality were related $\left(\mathrm{F}_{1,81}=4.26, \mathrm{P}=0.04\right)$. This finding indicates that the MSI scores of individuals with high moral identity decreased more $(\mathrm{M}=-8.58, \mathrm{SD}=2.82)$ than those of individuals with low moral identity $(\mathrm{M}=-5.87, \mathrm{SD}=3.31)$ and that individuals with high moral identity experienced higher degrees of moral dissonance (self-consistency). There were significant discrepancies in the MSI score differences between individuals with high moral identity and individuals with low moral identity under the condition that self-affirmation and morality were unrelated $\left(\mathrm{F}_{1,81}=4.55, \mathrm{P}=0.04\right)$. The MSI scores of individuals with high moral identity decreased less $(M=-5.29, S D=2.92)$ than those of individuals with low moral identity $(M=-2.38, S D=2.47)$, and individuals with high moral identity experienced lower degrees of moral dissonance. There were no significant discrepancies in the MSI score differences between individuals with high moral identity and individuals with low moral identity under the condition of no self-affirmations $\left(\mathrm{F}_{1,81}=0.07, \mathrm{P}=0.94\right)$. This outcome indicated that when there were no self-affirmation procedures, insignificant differences occurred in the degree of the decreases in the MSI scores between the participants of the high and low moral identity groups, and the participants did not experience different degrees of moral dissonance.

Table 1. The mean and standard deviation of self-affirmation and moral identity in the MSI scale

\begin{tabular}{cccccccc}
\hline & & \multicolumn{9}{c}{ Self-affirmation } \\
\cline { 3 - 7 } & & \multicolumn{2}{c}{ Moral related } & \multicolumn{2}{c}{ Moral unrelated } & \multicolumn{2}{c}{ No self-affirmation } \\
\cline { 3 - 8 } Moral Identity & low & -5.87 & SD & M & SD & M & SD \\
& high & -8.58 & 2.31 & -5.29 & 2.92 & -5.53 & 2.29 \\
& & 2.82 & -2.38 & 2.47 & -5.42 & 4.38 \\
\hline
\end{tabular}

Table 2. Effect of Self-affirmation and Moral Identity on the Change of MSI

\begin{tabular}{ccccccc}
\hline Source & $\begin{array}{c}\text { Sum of } \\
\text { squares }\end{array}$ & DF & Mean Square & F & P & Partial $\eta^{2}$ \\
\hline Self-affirmation & 160.12 & 2 & 80.06 & $8.28^{*}$ & 0.001 & 0.16 \\
$\begin{array}{c}\text { Moral Identity } \\
\text { Self-affirmation * Moral } \\
\text { Identity } \\
\text { Error }\end{array}$ & 0.21 & 1 & 0.21 & 0.02 & 0.88 & 0.0002 \\
& 109.76 & 2 & 54.88 & $54.88^{*}$ & 0.005 & 0.11 \\
\hline
\end{tabular}

MSI, Moral Self-Image.

Partial $\eta^{2}$ : small $=0.01$; medium $=0.06$; large $=0.14$ (Kittler, Menard, \& Phillips, 2007).

${ }^{*}$ Statistically significant at $\mathrm{P}<0.05$.

\section{Divergences between Self-affirmation and Self-consistency with Respect to Pro-social Behavior}

In the two-way between-subjects ANOVA, self-affirmation and moral identity were independent 
variables, while donation behavior was a dependent variable (means and standard deviations of donations are shown in Table 3). The findings revealed that the main effect of self-affirmation was significant $\left(\mathrm{F}_{2,79}=5.62, \mathrm{P}=0.005\right.$, partial $\left.\eta^{2}=0.11\right)$ and that the main effect of moral identity was insignificant $\left(\mathrm{F}_{1,79}=0.03, \mathrm{P}=0.086\right.$, partial $\left.\eta^{2}<0.001\right)$, as reported in Table 4 . The interactive influence was significant $\left(\mathrm{F}_{2,79}=4.876, \mathrm{P}=0.01\right.$, partial $\left.\eta^{2}=0.1\right)$. A simple effect analysis found marginally significant discrepancies in moral identity under the condition that morality and self-affirmation were related $\left(\mathrm{F}_{1,81}=3.73, \mathrm{P}=0.06\right)$. This outcome revealed that individuals with high moral identity experienced higher degrees of moral dissonance than individuals with low moral identity when self-affirmation and morality were related and therefore would engage in more pro-social behavior (self-consistency). There were significant discrepancies in moral identity under the condition that morality and self-affirmation were unrelated $\left(\mathrm{F}_{1,81}=4.38, \mathrm{P}=0.04\right)$. This outcome indicated that individuals with high moral identity experienced lower degrees of moral dissonance than individuals with low moral identity when self-affirmation and morality were unrelated and therefore would engage in less pro-social behavior (self-affirmation). There were no significant discrepancies in pro-social behavior when there were no self-affirmations $\left(\mathrm{F}_{1,81}<0.001, \mathrm{P}=0.97\right)$. This outcome indicated that there were no significant discrepancies in the subsequent pro-social behavior of individuals with high or low moral identity when there were no self-affirmations.

Table 3. The mean and standard deviation of self-affirmation and moral identity in the amount of donations

\begin{tabular}{cccccccc}
\hline & & \multicolumn{9}{c}{ Self-affirmation } \\
\cline { 3 - 8 } & & \multicolumn{2}{c}{ Moral related } & \multicolumn{2}{c}{ Moral unrelated } & \multicolumn{2}{c}{ No self-affirmation } \\
\cline { 3 - 8 } & M & SD & M & SD & M & SD \\
Moral & 6.33 & 3.33 & 6.14 & 3.59 & 6.27 & 3.31 \\
Identity & high & 8.78 & 2.01 & 3.38 & 3.01 & 6.21 & 3.17 \\
\hline
\end{tabular}

Table 4. Effect of self-affirmation and moral identity on the change of donation behavior

\begin{tabular}{ccccccc}
\hline Source & $\begin{array}{c}\text { Sum of } \\
\text { squares }\end{array}$ & df & $\begin{array}{c}\text { Mean } \\
\text { Square }\end{array}$ & F & P & Partial $\eta^{2}$ \\
\hline Self-affirmation & 109.17 & 2 & 54.58 & $5.62^{*}$ & 0.005 & 0.11 \\
Moral Identity & 0.30 & 1 & 0.302 & 0.03 & 0.86 & 0.0003 \\
Self-affirmation * Moral & 94.78 & 2 & 47.39 & $4.88^{*}$ & 0.010 & 0.1 \\
Identity & 767.77 & 79 & 9.72 & - & - & - \\
Error & & & & &
\end{tabular}

MSI, Moral Self-Image.

Partial $\eta^{2}$ : small $=0.01$; medium $=0.06$; large $=0.14$ (Kittler et al., 2007).

* Statistically significant at $\mathrm{P}<0.05$.

\section{Conclusion}

This study verified the regulatory function of moral identity on pro-social behaviors and employed experimental methods to probe how moral identity regulates pro-social behavior through self-affirmation. To a certain extent, using moral identity as a regulating variable clarified the differences between the self-affirmation theory and the self-consistency theory regarding pro-social behavior. In addition, through the moral self-image scale, the relationship between moral dissonance and pro-social behaviors was quantified. However, does moral identity continue to affect pro-social behavior in the absence of the self? This study did not address that question. Future studies could probe this issue further. For example, the personal or normative standards of judgment discussed by Stone and Cooper(Stone \& Cooper, 2001) could be applied, and future studies could investigate whether differences persist in the effect of moral identity on pro-social behavior under normative standards of judgment. 


\section{References}

[1] Henderson, P., \& Robert, P. (1992). Mental accounting and categorization. Organizational Behavioral and Human Decision Processes, 51, 92-117.

[2] Kittler, J. E., Menard, W., \& Phillips, K. A. (2007). Weight concerns in individuals with body dysmorphic disorder. Eating behaviors, 8(1), 115-120. doi: 10.1016/j.eatbeh.2006.02.006

[3] Reed, A., Aquino, K., \& Levy, E. (2007). Moral identity and judgments of charitable behaviors. Journal of Marketing, 71(1), 178-193. doi: 10.1509/jmkg.71.1.178

[4] Rong, Y., \& Haosheng, Y. (2014). The Black and White Metaphor Representation of Moral Concepts and Its Influence on Moral Cognition. Acta Psychologica Sinica, 46, 1331-1346.

[5] Steele, C. M., Spencer, S. J., \& Lynch, M. (1993). Silf-image Reslince and Dissonance - The Role of Affirmational Resources. Journal of personality and Social psychology, 64(6), 885-896. doi: 10.1037//0022-3514.64.6.885

[6] Xiaofu, P., Zhaojing, W., Fei, G., \& Ying, X. (2014). Irrational Consumption Bias of Windfall Gains and Hard-earned Money: Based on IAT and Evidence from an ERP Study. Advances in Psychological Science, 22, 596-605. doi: 10.3724/SP.J.1042.2014.00596 